Supplementary materials for

\title{
Metabolic engineering of Clostridium cellulovorans to improve butanol production by consolidated bioprocessing
}

Zhiqiang Wen ${ }^{\mathrm{a}}$, Rodrigo Ledesma-Amaro ${ }^{\mathrm{b}}$, Minrui Lu ${ }^{\mathrm{a}}$, Mingjie Jin ${ }^{\mathrm{a} *}$ Sheng Yang ${ }^{\mathrm{c}, \mathrm{d} *}$

a School of Environmental and Biological Engineering, Nanjing University of Science \& Technology, Nanjing, 210094, China

${ }^{b}$ Department of Bioengineering, Imperial College London, London SW7 2AZ, UK

${ }^{c}$ Key Laboratory of Synthetic Biology, CAS Center for Excellence in Molecular Plant Sciences, Shanghai Institute of Plant Physiology and Ecology, Chinese Academy of Sciences, Shanghai, 200032, China

${ }^{\mathrm{d}}$ Huzhou Center of Industrial Biotechnology, Shanghai Institutes of Biological Sciences, Chinese Academy of Sciences, Shanghai, 200032, China

*Corresponding authors: Sheng Yang, E-mail: syang@sibs.ac.cn; Mingjie Jin, E-mail: jinmingjie@njust.edu.cn 


\section{Supplementary Tables}

TABLE S1 Strains and plasmids used in this study.

\begin{tabular}{|c|c|c|}
\hline Strains or plasmids & Description of genotype & Source or reference \\
\hline \multicolumn{3}{|l|}{ Strains } \\
\hline \multirow[t]{2}{*}{ E. coli $\mathrm{DH} 5 \alpha$} & Commercial transformation host & GIBCO BRL, Life \\
\hline & & Technologies \\
\hline \multirow[t]{3}{*}{ E. coli ER2275 } & Strain used to pre-methylate plasmids for & New England Biolabs \\
\hline & electron-transformation in C. acetobutylicum & \\
\hline & ATCC 824 and C. cellulovorans DSM 743B & \\
\hline C. acetobutylicum ATCC 824 & Wild type & ATCC \\
\hline C. cellulovorans DSM 743B & Wild type & DSMZ \\
\hline $743 \mathrm{~B}\left(\mathrm{pXY} 1-\mathrm{P}_{t h l}\right)$ & $743 \mathrm{~B} / \mathrm{pXY} 1-\mathrm{P}_{t h l}$ & 1 \\
\hline $743 \mathrm{~B}\left(\mathrm{pXY} 1-\mathrm{P}_{t h l}-a d h E 1\right)$ & $743 \mathrm{~B} / \mathrm{pXY} 1-\mathrm{P}_{t h l}-a d h E 1$ & 2 \\
\hline 743B(pXY1-P $\left.{ }_{t h l}-t e r\right)$ & 743B/pXY1-P thl-ter & This study \\
\hline 743B(pXY1-P $\left.{ }_{t h l}-a d h E 1-\mathrm{P}_{t h l}-t e r\right)$ & $743 \mathrm{~B} / \mathrm{pXY} 1-\mathrm{P}_{t h l}-c t f A B-a d c$ & This study \\
\hline 743B(pXY1-P $\left.{ }_{t h l}-a d h E 1-\mathrm{P}_{t h l}-\mathrm{CAT} 1\right)$ & 743B/pXY1-P ${ }_{t h l}-a d h E 1-\mathrm{P}_{t h l}-\mathrm{CAT} 1$ & This study \\
\hline 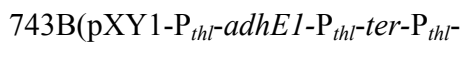 & 743B/pXY1- $\mathrm{P}_{t h l}-a d h E 1-\mathrm{P}_{t h l}-t e r-\mathrm{P}_{t h l}-\mathrm{CAT} 1$ & This study \\
\hline \multicolumn{3}{|l|}{ CAT1) } \\
\hline $743 \mathrm{~B} \Delta x y l R$ & 743B $x y l R::$ intron & This study \\
\hline $743 \mathrm{~B} \Delta x y l R\left(\mathrm{pXY} 1-\mathrm{P}_{t h l}-x y l R\right)$ & 743B $x y l R::$ intron/pXY1-P $\mathrm{P}_{t h l}-x y l R$ & This study \\
\hline 743B $\triangle a r a R$ & 743B araR::intron & This study \\
\hline 743B $\Delta a r a R\left(\mathrm{pXY} 1-\mathrm{P}_{t h l}-a r a R\right)$ & 743B $\operatorname{araR}::$ intron $/ \mathrm{pXY} 1-\mathrm{P}_{t h l}-\operatorname{araR}$ & This study \\
\hline 743B $\triangle x y l R \triangle a r a R$ & 743B xylR::intron-araR::intron & This study \\
\hline $743 \mathrm{~B}\left(\mathrm{pXY} 1-\mathrm{P}_{t h l^{-}} x y l T\right)$ & 743B/pXY1- ${ }_{t h l}-x y l T$ & This study \\
\hline 743B $\Delta x y l R \Delta a r a R-\left(\mathrm{pXY} 1-\mathrm{P}_{t h l}-x y l T\right)$ & $\begin{array}{l}\text { 743B } x y l R:: \text { intron-araR::intron/pXY1-P }{ }_{t h l^{-}} \\
x y l T\end{array}$ & This study \\
\hline 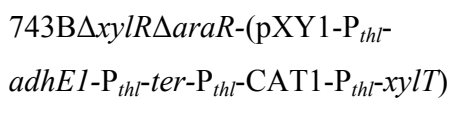 & 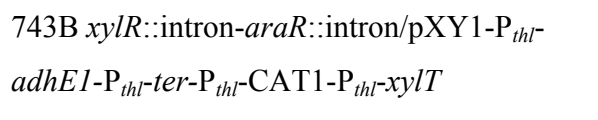 & This study \\
\hline \multicolumn{3}{|l|}{ Plasmids } \\
\hline pANS1 & $\Phi 3 T I$ gene, p15A origin; Spec $^{\mathrm{r}}$ & 3 \\
\hline $\mathrm{pXY} 1-\mathrm{P}_{t h l}$ & $\begin{array}{l}\text { Amp }^{\mathrm{r}}, \mathrm{MLS}^{\mathrm{r}}, \mathrm{pCB} 102 \mathrm{ORI}, \mathrm{ColE} 1 \text { origin, } T h l \\
\left(C A \_ \text {_c2873) promoter region of } C \text {. }\right. \\
\text { acetobutylicum; E. coli-C. cellulovorans } \\
\text { shuttle vector for expressing genes in } C \text {. } \\
\text { cellulovorans }\end{array}$ & 4 \\
\hline pWJ1-araR & $\begin{array}{l}\text { Derived from } \mathrm{pWJ} 1^{5} \text { for intron insertion in } C \text {. } \\
\text { cellulovorans araR at } 264 / 265 \mathrm{nt}\end{array}$ & This study \\
\hline pWJ1-xylR & $\begin{array}{l}\text { Derived from } \mathrm{pWJ} 1^{5} \text { for intron insertion in } C \text {. } \\
\text { cellulovorans } x y l R \text { gene at } 987 / 988 \mathrm{nt}\end{array}$ & This study \\
\hline $\mathrm{pXY} 1-\mathrm{P}_{t h l}-\operatorname{araR}$ & $\begin{array}{l}\text { Derived from pXY1-P }{ }_{t h l} \text {, with araR } \\
\text { (Clocel_1253) gene complementation }\end{array}$ & This study \\
\hline
\end{tabular}




\begin{tabular}{|c|c|c|}
\hline $\mathrm{pXY} 1-\mathrm{P}_{t h l}-x y l R$ & $\begin{array}{l}\text { Derived from } \mathrm{pXY} 1-\mathrm{P}_{t h l} \text {, with } x y l R \\
\text { (Clocel_0594) gene complementation }\end{array}$ & This study \\
\hline $\mathrm{pXY} 1-\mathrm{P}_{t h l}-t e r$ & $\begin{array}{l}\text { Derived from } \mathrm{pXY} 1-\mathrm{P}_{t h l}, \text { with a synthesized } \\
\text { ter gene (codon optimization) from }\end{array}$ & This study, ${ }^{6}$ \\
\hline & Treponema denticola overexpression & \\
\hline $\mathrm{pXY} 1-\mathrm{P}_{t h l}-x y l T$ & $\begin{array}{l}\text { Derived from } \mathrm{pXY} 1-\mathrm{P}_{t h l}, \text { with } x y l T \\
\left(C A_{-} \text {C1345) from C. acetobutylicum }\right. \\
\text { overexpression }\end{array}$ & This study \\
\hline $\mathrm{pXY} 1-\mathrm{P}_{t h l}-C A T 1$ & $\begin{array}{l}\text { Derived from } \mathrm{pXY} 1-\mathrm{P}_{\text {thl}}, \text { withCAT1 } \\
\left(C T K \_C 06520\right) \text { gene from C. tyrobutyricum }\end{array}$ & This study \\
\hline & DSM 2637 overexpression & \\
\hline $\mathrm{pXY} 1-\mathrm{P}_{t h l}-a d h E 1$ & $\begin{array}{l}\text { Derived from } \mathrm{pXY} 1-\mathrm{P}_{t h l}, \text { with adhE1 } \\
\left(C A \_ \text {0162) gene from C. acetobutylicum }\right. \\
\text { overexpression }\end{array}$ & This study \\
\hline $\mathrm{pXY} 1-\mathrm{P}_{t h l}-a d h E 1-\mathrm{P}_{t h l}-t e r$ & $\begin{array}{l}\text { Derived from pXY1- } \mathrm{P}_{t h l}-a d h E 1 \text {, with } \mathrm{P}_{t h l} \text {-ter } \\
\text { cassette expression }\end{array}$ & This study \\
\hline $\mathrm{pXY} 1-\mathrm{P}_{t h l}-a d h E 1-\mathrm{P}_{t h l}-C A T 1$ & $\begin{array}{l}\text { Derived from } \mathrm{pXY} 1-\mathrm{P}_{t h l^{-}} a d h E 1, \text { with } \mathrm{P}_{t h l^{-}} \\
C A T 1 \text { cassette expression }\end{array}$ & This study \\
\hline 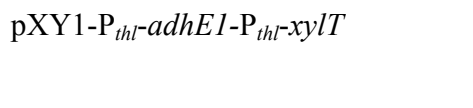 & $\begin{array}{l}\text { Derived from } \mathrm{pXY} 1-\mathrm{P}_{t h l}-a d h E 1, \text { with } \mathrm{P}_{t h l}-x y l T \\
\text { cassette expression }\end{array}$ & This study \\
\hline $\mathrm{pXY} 1-\mathrm{P}_{t h l}-a d h E 1-\mathrm{P}_{t h l}-t e r-\mathrm{P}_{t h l}-C A T 1$ & $\begin{array}{l}\text { Derived from } \mathrm{pXY} 1-\mathrm{P}_{t h l}-a d h E 1-\mathrm{P}_{t h l^{-} t e r, \text { with }} \\
\mathrm{P}_{t h l^{-} C A T 1 \text { cassette expression }}\end{array}$ & This study \\
\hline 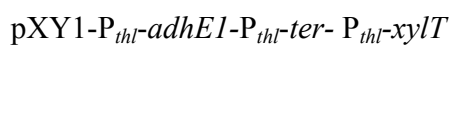 & $\begin{array}{l}\text { Derived from } \mathrm{pXY} 1-\mathrm{P}_{t h l}-a d h E 1-\mathrm{P}_{t h l} \text {-ter, with } \\
\mathrm{P}_{t h l} \text {-xylT cassette expression }\end{array}$ & This study \\
\hline $\begin{array}{l}\mathrm{pXY} 1-\mathrm{P}_{t h l^{-}} a d h E 1-\mathrm{P}_{t h l^{-}} C A T 1-\mathrm{P}_{t h l^{-}} \\
x y l T\end{array}$ & $\begin{array}{l}\text { Derived from } \mathrm{pXY} 1-\mathrm{P}_{t h l}-a d h E 1-\mathrm{P}_{t h l}-C A T 1 \text {, } \\
\text { with } \mathrm{P}_{t h l}-x y l T \text { cassette expression }\end{array}$ & This study \\
\hline $\mathrm{pXY} 1-\mathrm{P}_{t h l^{-}}-a d h E 1-\mathrm{P}_{t h l^{-}}$ter- $\mathrm{P}_{t h l^{-}}$ & Derived from $\mathrm{pXY} 1-\mathrm{P}_{t h l^{-}}-a d h E 1-\mathrm{P}_{t h l^{-}}$ter $-\mathrm{P}_{t h l^{-}}$ & This study \\
\hline$C A T 1-\mathrm{P}_{t h l}-x y l T$ & $C A T 1$, with $\mathrm{P}_{t h l^{-}} x y l T$ cassette expression & \\
\hline
\end{tabular}

ATCC, American Type Culture Collection, America; DSMZ, Deutsche Sammlung von Mikroorganismen und Zellkulturen, Germany; Spe ${ }^{\mathrm{r}}$, spectinomycin resistance; $\mathrm{Amp}^{\mathrm{r}}$, ampicillin resistance; MLS ${ }^{\mathrm{r}}$, macrolidelincosamide-streptogramin resistance; pCB102 ORI, gram-positive origin of replication from Clostridium butyricum; Thl, thiolase. 
TABLE S2 Primers used in this study.

\begin{tabular}{|c|c|c|}
\hline Primer name & Sequence (5'-3') & Description \\
\hline EBS universal & CGAAATTAGAAACTTGCGTTCAGTAAAC & Targetron primer ${ }^{\mathrm{a}}$ \\
\hline & CCGCTCGAGATAATTATCCTTACACGACA & ara $R$ Targetron nrimera \\
\hline IBS & GCAAAGTGCGCCCAGATAGGGTG & \\
\hline \multirow{3}{*}{$\begin{array}{l}\text { araR-264|265s - } \\
\text { EBS1d }\end{array}$} & AGATTGTACAAATGTGGTGATAACAGATA & \multirow{3}{*}{ araR Targetron primer ${ }^{\mathrm{a}}$} \\
\hline & AGTCAGCAAAACTAACTTACCTTTCTTTG & \\
\hline & $\mathrm{T}$ & \\
\hline & TGAACGCAAGTTTCTAATTTCGGTTTCGT & \multirow{2}{*}{ araR Targetron primer ${ }^{\mathrm{a}}$} \\
\hline EBS2 & GTCGATAGAGGAAAGTGTCT & \\
\hline araR_1-20 & ATGTTTAAAAAACCTATAGT & $\begin{array}{l}\text { Forward primer inside araR from } 1 \text { to } 20 \text { base, } \\
\text { Ch(intron-sF) }\end{array}$ \\
\hline araR_410-420 & TTGGTTTACCTTTTGATGTATG & $\begin{array}{l}\text { Revise primer inside } a r a R \text { from } 410 \text { to } 430 \text { base, } \\
\text { Ch(intron-sR) }\end{array}$ \\
\hline $\operatorname{araR~F}$ & $\begin{array}{l}\text { AAATTTAGGAGGTTAGTTAGAGGATCCAT } \\
\text { GTTTAAAAAACCTATAGTTTTAAACGGTA } \\
\text { A }\end{array}$ & $\begin{array}{l}\text { Forward primer for araR clone from } 743 \mathrm{~B} \\
\text { chromosome and assembly in } \mathrm{pXY} 1-\mathrm{P}_{t h l^{-}} \text {araR }\end{array}$ \\
\hline $\arg \mathrm{R} R$ & TTGTAAAACGACGGCCAGTGAATTCCCGG & \multirow{2}{*}{$\begin{array}{l}\text { Reverse primer for araR clone from } 743 \mathrm{~B} \\
\text { chromosome and assembly in } \mathrm{pXY} 1-\mathrm{P}_{t h l^{-}} \text {araR }\end{array}$} \\
\hline $\operatorname{arak} \mathrm{K}$ & GTCATTGATATATATTGATACATGATTTCT & \\
\hline xylR-987|988s- & CCGCTCGAGATAATTATCCTTAGTAATCG & \multirow{2}{*}{$x y l R$ Targetron primer ${ }^{\mathrm{a}}$} \\
\hline IBS & GCGGGGTGCGCCCAGATAGGGTG & \\
\hline & AGATTGTACAAATGTGGTGATAACAGATA & \multirow[b]{2}{*}{$x y l R$ Targetron primer ${ }^{\mathrm{a}}$} \\
\hline EBS1d & $\begin{array}{l}\text { AGTCGGCGGGGATAACTTACCTTTCTTTG } \\
\mathrm{T}\end{array}$ & \\
\hline $\begin{array}{l}\text { xylR-987|988s- } \\
\text { EBS2 }\end{array}$ & $\begin{array}{l}\text { TGAACGCAAGTTTCTAATTTCGATTATTA } \\
\text { CTCGATAGAGGAAAGTGTCT }\end{array}$ & $x y l R$ Targetron primer ${ }^{\mathrm{a}}$ \\
\hline xylR_804-823 & AGCACTATTAAAAACATATA & $\begin{array}{l}\text { Forward primer inside } x y l R \text { from } 804 \text { to } 823 \text { base, } \\
\text { Ch(intron-sF) }\end{array}$ \\
\hline xylR_1154-1173 & CTATATAGTTATATTGTTAT & $\begin{array}{l}\text { Revise primer inside } x y l R \text { from } 1154 \text { to } 1173 \\
\text { base, } \mathrm{Ch} \text { (intron-sR) }\end{array}$ \\
\hline xylR F & $\begin{array}{l}\text { AAATTTAGGAGGTTAGTTAGAGGATCCTT } \\
\text { GACAAAAATAAAAAAGTTAGATCAAGAA } \\
\text { AC }\end{array}$ & $\begin{array}{l}\text { Forward primer for } x y l R \text { clone from } 743 \mathrm{~B} \\
\text { chromosome and assembly in } \mathrm{pXY} 1-\mathrm{P}_{t h l^{-}} x y l R\end{array}$ \\
\hline xylR R & $\begin{array}{l}\text { TTGTAAAACGACGGCCAGTGAATTCCCGG } \\
\text { GCTATATAGTTATATTGTTATTAAAAAAT } \\
\text { A }\end{array}$ & $\begin{array}{l}\text { Reverse primer for } x y l R \text { clone from } 743 \mathrm{~B} \\
\text { chromosome and assembly in } \mathrm{pXY} 1-\mathrm{P}_{t h l^{-}} x y l R\end{array}$ \\
\hline adhE1 F & $\begin{array}{l}\text { AAATTTAGGAGGTTAGTTAGAGGATCCCC } \\
\text { ATGAAAGTCACAACAGTAAAGGAATTAG } \\
\text { AT }\end{array}$ & $\begin{array}{l}\text { Forward primer for gene } a d h E 1 \text { clone from } C \text {. } \\
\text { acetobutylicum genome and assembly in plasmid } \\
\text { pXY1-P }{ }_{t h l}-a d h E 1\end{array}$ \\
\hline adhE1 R & $\begin{array}{l}\text { GACGTTGTAAAACGACGGCCAGTGAATTC } \\
\text { CCTTAAGGTTGTTTTTTAAAACAATTTATA }\end{array}$ & $\begin{array}{l}\text { Reverse primer for gene adhE1 clone from } C \text {. } \\
\text { acetobutylicum genome and assembly in plasmid } \\
\text { pXY1- }{ }_{t h l} \text {-adhE1 }\end{array}$ \\
\hline ter $\mathrm{F}$ & $\begin{array}{l}\text { GTTGTAAAACGACGGCCAGTGAATTCCCG } \\
\text { GGTTATATTCTATCAAATCTTTCAАCTTCA }\end{array}$ & $\begin{array}{l}\text { Forward primer for a codon optimized gene ter } \\
\text { clone assembly in plasmid } \mathrm{pXY} 1-\mathrm{P}_{t h l}-t e r\end{array}$ \\
\hline
\end{tabular}




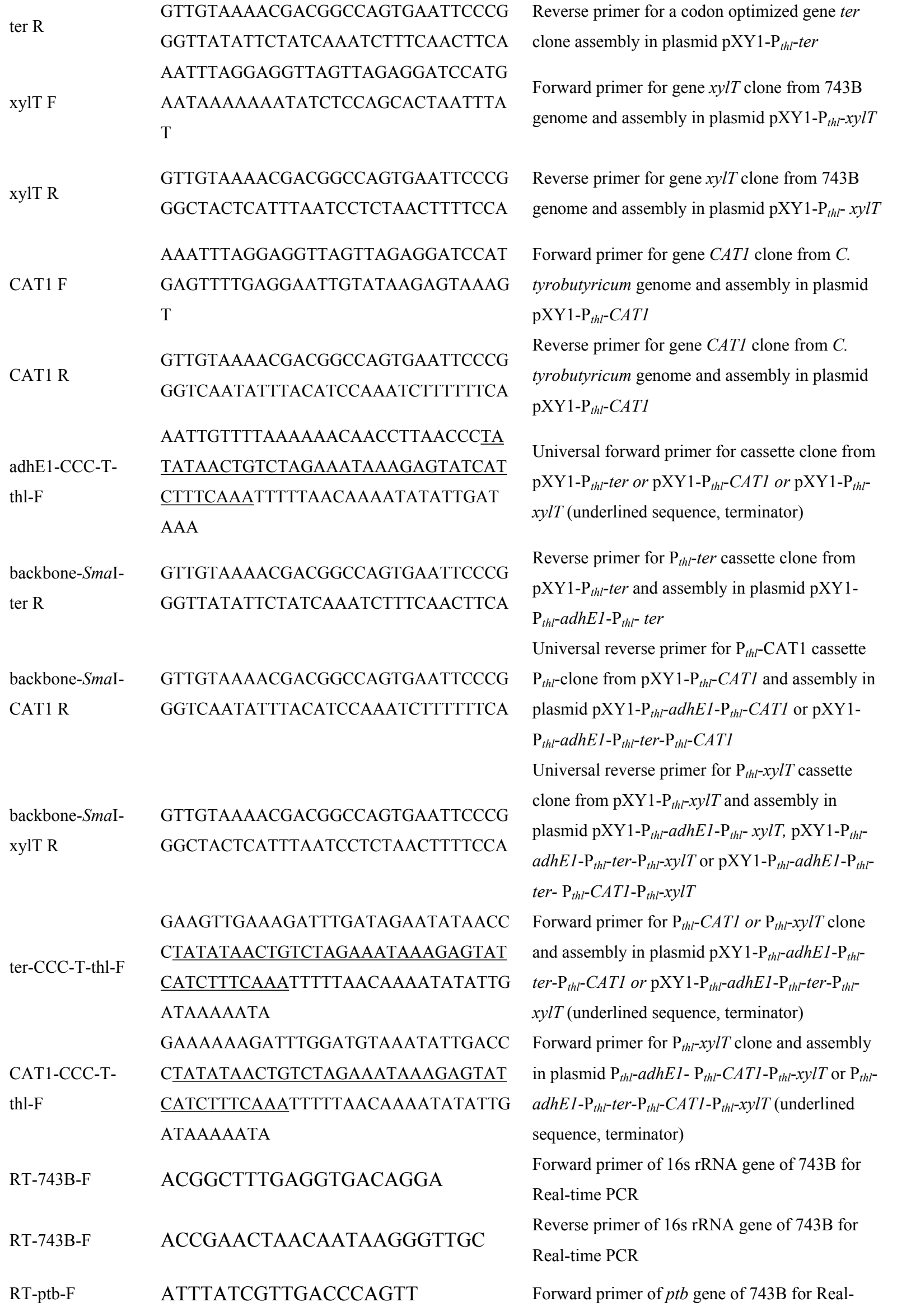


time PCR

RT-ptb-R

CCATCTAATGCGTTGTTT

RT-bk-F

RT-bk-R
TGTTAATACAACTGGTGC TGTTAATACAACTGGTGC
Reverse primer of $p t b$ gene of 743B for Real-time PCR

Forward primer of $b k$ gene of 743B for Real-time PCR

Reverse primer of $b k$ gene of 743B for Real-time PCR

a Designed by on-line tools (www.clostron.com) 


\section{Supplementary figures}
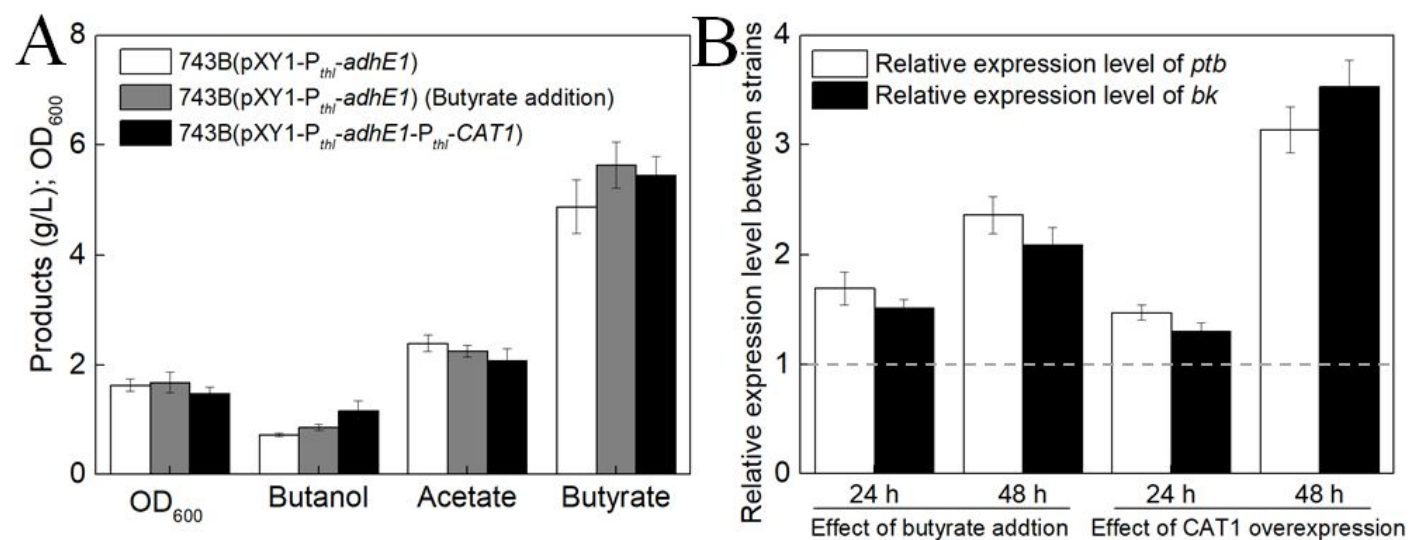

Figure S1 effect of butyrate addition and CAT1 overexpression on PTB-BK channel. (A), phenotype comparison among $743 \mathrm{~B}\left(\mathrm{pXY} 1-\mathrm{P}_{t h l}\right.$-adhE1-P $\mathrm{P}_{t h l}$-CAT1) as well as $743 \mathrm{~B}\left(\mathrm{pXY} 1-\mathrm{P}_{t h l}\right.$-adhE1) with and without butyrate addition; (B), Change of $p t b / b k$ transcriptional level between $743 \mathrm{~B}\left(\mathrm{pXY} 1-\mathrm{P}_{t h l}\right.$-adhE1) with and without butyrate addition; Change of $p t b / b k$ transcriptional level between $743 \mathrm{~B}\left(\mathrm{pXY} 1-\mathrm{P}_{t h l}-\right.$ adhE1- $\left.\mathrm{P}_{t h l}-\mathrm{CAT} 1\right)$ and 743B(pXY1- $\mathrm{P}_{t h l^{-}}$ adhE1).
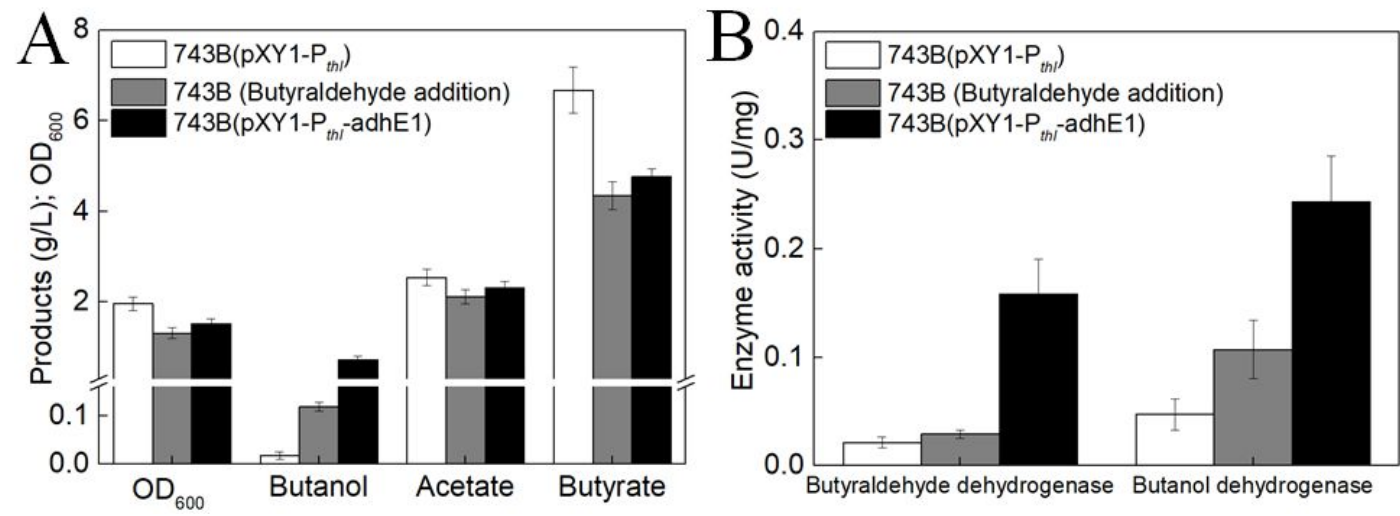

Figure S2 Butyraldehyde dehydrogenase as well as butanol dehydrogenase activities in WT cultures with and without addition of butyraldehyde as well as in the recombinant strain $743 B\left(p X Y 1-P_{t h r} r^{-a d h E 1}\right)$. (A), phenotype comparison; (B) Butyraldehyde dehydrogenase as well as butanol dehydrogenase activities comparison. 


\section{Supplementary sequence}

Codon optimized sequence of ter

ATGATAGTAAAACCAATGGTTAGAAATAATATTTGTTTAAATGCTCATCCACAAGGAT GTAAAAAAGGAGTAGAAGATCAAATTGAATATACTAAAAAGAGAATAACTGCTGAA GTTAAAGCAGGAGCTAAAGCACCTAAAAATGTATTAGTTTTAGGATGTAGTAATGGT TATGGTTTAGCATCTAGAATTACTGCAGCTTTTGGATATGGAGCTGCAACAATAGGAG TTAGTTTTGAAAAAGCAGGTAGTGAAACTAAATATGGTACTCCAGGATGGTATAATA ATTTAGCATTTGATGAAGCTGCTAAAAGAGAAGGATTATATAGTGTAACAATTGATG GAGATGCATTTAGTGATGAAATTAAAGCACAAGTAATTGAAGAAGCTAAGAAAAAA GGTATTAAATTTGATCTAATAGTATATTCTTTAGCTTCACCAGTAAGAACTGATCCTG ATACTGGAATAATGCATAAATCAGTATTAAAACCATTTGGTAAAACATTTACTGGAA AAACAGTTGATCCATTTACAGGAGAATTAAAAGAAATTAGTGCTGAGCCAGCTAATG ATGAAGAAGCAGCTGCAACAGTTAAAGTAATGGGAGGAGAAGATTGGGAAAGATGG ATAAAACAATTATCAAAAGAAGGTTTACTTGAAGAAGGTTGTATAACTTTAGCTTATT CTTATATAGGACCAGAAGCTACTCAAGCTTTATATAGAAAAGGAACAATAGGAAAAG CTAAAGAACATTTAGAAGCTACAGCACATAGATTAAATAAGGAAAATCCATCAATAA GAGCATTTGTATCAGTTAATAAGGGATTAGTAACTAGAGCTAGTGCAGTAATACCTGT AATTCCTTTATATTTAGCATCATTATTTAAAGTAATGAAAGAAAAAGGAAATCATGAA GGATGTATTGAACAAATAACAAGACTTTATGCTGAAAGATTATATAGAAAAGATGGT ACTATACCAGTAGATGAAGAAAATAGAATAAGAATAGATGATTGGGAATTAGAAGA AGATGTACAAAAAGCTGTTAGTGCATTAATGGAAAAAGTTACTGGAGAAAATGCTGA ATCATTAACTGATTTAGCTGGATATAGACATGATTTCTTAGCTTCAAATGGATTTGAT GTAGAAGGAATTAATTATGAAGCTGAAGTTGAAAGATTTGATAGAATATAA 


\section{REFERENCES}

(1) Wen, Z., Minton, N. P., Zhang, Y., Li, Q., Liu, J., Jiang, Y., and Yang, S. (2017) Enhanced solvent production by metabolic engineering of a twin-clostridial consortium. Metab. Eng., 39, 3848.

(2) Wen, Z., Ledesma-Amaro, R., Lin, J., Jiang, Y., and Yang, S. (2019) Improved n-butanol production from Clostridium cellulovorans by integrated metabolic and evolutionary engineering. Appl. Environ. Microbiol., 85.

(3) Mermelstein, L., and Papoutsakis, E. (1993) In vivo methylation in Escherichia coli by the Bacillus subtilis phage phi 3T I methyltransferase to protect plasmids from restriction upon transformation of Clostridium acetobutylicum ATCC 824, Appl. Environ. Microbiol., 59, 10771081.

(4) Zhang, N., Shao, L., Jiang, Y., Gu, Y., Li, Q., Liu, J., Jiang, W., and Yang, S. (2015) I-SceImediated scarless gene modification via allelic exchange in Clostridium. J. Microbiol. Methods, 108, 49-60.

(5) Xiao, H., Gu, Y., Ning, Y., Yang, Y., Mitchell, W. J., Jiang, W., and Yang, S. (2011) Confirmation and elimination of xylose metabolism bottlenecks in glucose phosphoenolpyruvatedependent phosphotransferase system-deficient Clostridium acetobutylicum for simultaneous utilization of glucose, xylose, and arabinose, Appl. Environ. Microbiol., 77, 7886-7895.

(6) Shen, C. R., Lan, E. I., Dekishima, Y., Baez, A., Cho, K. M., and Liao, J. C. (2011) Driving forces enable high-titer anaerobic 1-butanol synthesis in Escherichia coli. Appl. Environ. Microbiol., 77, 2905-2915. 